\title{
How trinucleotide repeats may function
}

The hereditary neurodegenerative diseases marked by repetitive genetic elements may arise from interaction between the mutated proteins and others specific to the brain.

FIVE hereditary neurodegenerative diseases are now known to involve expansions of the trinucleotide CAG within the susceptibility genes, resulting in an abnormally long stretch of polyglutamine within the encoded proteins. Each of these diseases involves a progressive and often fatal loss of specific populations of neurons; the best-known is Huntington's disease (HD), while the others include spinocerebellar ataxia type 1 (SCA1), Machado-Joseph disease (SCA3), spinobulbar muscular atrophy (SBMA) and dentatorubral and pallidolyusian atrophy (DRPLA). Why do expanded stretches of polyglutamine appear to be so damaging?

Two important contributions to answering this question are to be published in Nature on 23 November. As chance will have it, the authors will also be presenting an account of their findings next Monday (13 November) at the annual meeting of the Society of Neuroscience at San Diego, California. For the benefit of those who will not be there, or who may read of these important developments elsewhere, what follows is a brief summary of the findings.

A distinctive feature of all these diseases is a phenomenon known as genetic anticipation, whereby the symptoms appear at earlier ages and with greater severity in successive generations. It is now clear that anticipation is due to the instability of the amplified DNA sequence; as it passes through the germline (particularly the paternal line, which undergoes more cell divisions per generation than the maternal line), the number of repeats increases. A recent study suggests that the repeats form hairpin structures that disrupt normal DNA replication (Cell 81, 533-540; 1995); once the number of uninterrupted repeats exceeds a critical threshold, it may form a stable hairpin, thereby leading to a vicious circle of replication errors and further expansion.

To explain the amplification of trinucleotide repeats is one thing, but to understand - and ultimately to prevent - the consequences is another matter. It now appears that polyglutamine expansion renders the altered proteins not merely useless but actively harmful (in genetic parlance, a gain-of-function mutation); each of the five syndromes is genetically dominant, and in cases where the loss-of-function phenotype is known, it does not resemble the disease pathology; loss of huntingtin, the HD gene product, causes embryonic lethality (Cell 81, 811-823; 1995; Science 269, 407-410; 1995) while the SBMA gene encodes the androgen receptor, whose absence leads not to neurodegeneration but to testicular feminization. Moreover, a transgenic mouse with an expanded SCA-1 gene develops Purkinje cell degeneration, whereas the normal gene has no effect (Cell 82, 937-948: 1995).

If expanded glutamine repeats kill neurons, how do they do it? Polyglutamine occurs in several transcription factors, but an effect on transcription seems unlikely, given that at least some of the mutant proteins are cytoplasmic rather than nuclear (Nature Genet. 10, 3-4; 1995). Another possibility is that polyglutamine may lead to the formation of insoluble aggregates; but there is little evidence that any of the proteins accumulate to unusually high levels in affected tissues. Many suspect that the expansion of the polyglutamine may lead to new and pathological interactions with a target protein, hitherto unidentified.

Now, Chris Ross and his colleagues at Johns Hopkins University School of Medicine have identified a protein they term HAP-1 (for huntingtin-associated protein 1), which binds to the mutant form of huntingtin both in a yeast two-hybrid screen and in transfected cells. It is not yet known whether it also binds to huntingtin in the brains of affected individuals - a crucial question - but the restriction of its expression to the brain could explain why the mutated huntingtin protein kills brain cells while leaving other tissues unscathed. Most important, the strength of binding between HAP-1 and huntingtin depends on the number of glutamines; wild-type huntingtin, with 19-22 repeats, binds only weakly, whereas the mutant forms bind more strongly as the length of the polyglutamine stretch increases from 44 to 82 repeats. Length is probably not the only factor, because atrophin-1 (the DRPLA gene product) contains 21 glutamines but shows no detectable binding. Whether longer, pathological forms of atrophin-1 (Nature Genet. 6, 9-13; 1994) can bind HAP-1 remains unknown.

The case for HAP-1 involvement in HD is still circumstantial, and will remain so until there is hard genetic evidence. Optimists may hope that a HAP-1 knockout mouse will succumb to progressive dementia and loss of neurons, but it seems equally possible that HAP-1 is activated, rather than inhibited, by polyglutamines. The sequence of HAP-1 offers no clue to its function, although it has at least two relatives that may also prove to be important. In short, the questions are clear but many months of hard work will be needed to answer them.

The second new study, by Jean-Louis Mandel and colleagues (INSERM, Stras- bourg), strongly suggests that all the neurodegenerative diseases arise from a common mechanism involving a change in protein conformation. The authors have obtained a monoclonal antibody (1C2), which binds polyglutamine stretches both in huntingtin and in the SCA1 and SCA3 gene-products. Like the association between HAP-1 and huntingtin, the strength of $1 \mathrm{C} 2$ binding is correlated with the number of glutamine repeats, being largely specific to the mutant forms of these proteins. This may be due to the formation of a so-called 'polar zipper' structure, in which $\beta$-strands interact via hydrogen bonding (Proc. natn. Acad. Sci. U.S.A. 92, 6509-6513; 1995). Perhaps when it reaches a certain length, the polypeptide strand can fold back on itself to form a stable hairpin; crystal structures will be awaited with interest.

The two new studies have several immediate implications. First, they offer the prospect of a biochemical assay for the pathogenetic process. It should be simple enough to screen for small molecules that block the interaction, and the fact that glutamine repeats are not highly conserved in evolution and may therefore have no essential function is encouraging, as is the likelihood that the HAP-1 interaction involves an abnormal structural conformation; it should in principle be possible to block it without disrupting any normal physiological process.

The 1C2 antibody also offers a strategy for the identification of further trinucleotide disease genes, and indeed two other diseases (SCA2 and autosomal dominant cerebellar ataxia type II), as yet uncharacterized at the molecular level, also involve the appearance of proteins that react with $1 \mathrm{C} 2$ and so presumably contain expanded polyglutamine repeats. Seven hereditary ataxias have been described, and as they all show genetic anticipation, it seems likely that all will involve the same mechanism. Because their pathological manifestations are poorly defined and change with each generation, a biochemical method of diagnosis will be invaluable for isolating the genes involved. But a bigger prize may lie ahead. Bipolar disorder (manic depression) and schizophrenia, which are much more common than the hereditary ataxias, both show genetic anticipation (Trends Neurosci. 16, $254-260 ; 1993)$, although attempts to map susceptibility loci have been inconclusive. Neither condition is known to involve neurodegeneration, but this may occur in subpopulations of neurons that have yet to be recognized. A positive result would indeed be a spectacular payoff. Charles Jennings 